\title{
R-phase stabilization in ultra-fine grain NiTi wires after mechanical cycling
}

\author{
A. Condó ${ }^{1}$, Ch. Somsen ${ }^{2}$, J. Olbricht ${ }^{2}$, G. Eggeler ${ }^{2}$, A. Dlouhý ${ }^{3, a}$ \\ ${ }^{1}$ Centro Atómico Bariloche, Av. Bustillo 9.500, 8400 S.C. de Bariloche, Argentina \\ ${ }^{2}$ Ruhr-Universität Bochum, Institut für Werkstoffe, Universitätsstr. 150, 44801 Bochum, Germany \\ ${ }^{3}$ Academy of Sciences CR, Institute of Physics of Materials, Zizkova 22, 61662 Brno, Czech Republic
}

\begin{abstract}
Loading-unloading cycles were applied in order to induce forward and reverse martensitic transformations B2 <-> (R) <-> B19' in ultra-fine grained NiTi (50.9 at\% Ni) wires. Stress-strain hysteresis loops change with increasing number of cycles. It has been observed that the upper plateau stress, which corresponds to the forward transformation induced in the loading part of the cycle, decreases considerably during mechanical cycling. In contrast, an only moderate drop of the lower plateau stresses characterizes the reverse transformation before the hysteresis loops reach their saturated shape. Moreover, a permanent plastic strain of the order of $1 \%$ accumulates before the hysteresis loops saturate. An extensive transmission electron microscopy investigation on the nano-grain scale has been performed in the present study in order to account for the observed hysteresis loop evolution. A representative sample of the selected area diffraction $(\mathrm{SAD})$ rings was evaluated for both, the as received state of the wire before the fatigue experiment and the mechanically cycled material. Results of the SAD analysis suggest that the loading cycles stabilize the R phase. However, no evidence was found for retained B19' phase after cyclic loading. Some consequences of these microstructural findings are discussed.
\end{abstract}

\section{Introduction}

A stable mechanical behaviour is a prerequisite for successful applications of NiTi based shape memory alloys [1]. Nevertheless, an asymmetric evolution of hysteresis loops have been frequently observed during mechanical cycling of NiTi based samples [2-4]. Figure 1 shows a typical example in which the loading plateau stress decreases with increasing number of superelastic cycles. The unloading plateau is much less affected by mechanical cycling. The hysteresis loops shown in Fig. 1 were obtained for Ni-rich NiTi (50.9 at $\%$ Ni) ultra-fine grain wires tested at $60^{\circ} \mathrm{C}\left(\mathrm{A}_{\mathrm{f}}\right.$ being about $\left.5^{\circ} \mathrm{C}\right)$. The understanding of a microstructural development governing the indicated variations of functional properties is far from being complete [3]. It has been suggested that dislocations generated [2, 3, 5] and/or particles sheared [2] by the B19' martensite may contribute to the observed macroscopic effects. Recent experiments with cold rolled and straight annealed NiTi ribbons provided some transmission electron microscopy (TEM) evidence showing that microstructural changes associated with

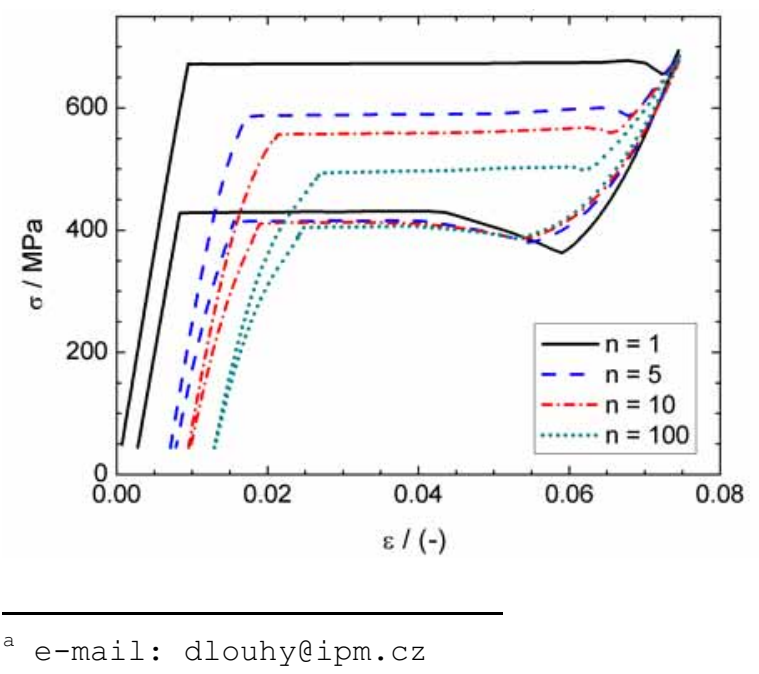

Fig. 1. Evolution of hysteresis loops with increasing number of superelastic cycles in Ti-50.9at $\% \mathrm{Ni}$ wire at $60^{\circ} \mathrm{C}$.

This is an Open Access article distributed under the terms of the Creative Commons Attribution-Noncommercial License (http://creativecommons.org/licenses/by-nc/3.0/), which permits unrestricted use, distribution, and reproduction in any noncommercial medium, provided the original work is properly cited. 
the functional fatigue involve a stabilization of R phase and even a stabilization of B19' crystallites in the parent cubic B2 austenite phase [5]. A typical size of martensitic regions retained in the unloading B2 state of the ribbons after cycling was reported well below $20 \mathrm{~nm}$ [5].

These findings support a more comprehensive view of dislocations induced by cycling and martensitic phases stabilized by dislocation stress fields [3]. However, the experiments performed with the NiTi ribbons revealed that even the initial ribbon microstructure was inhomogeneous consisting of ultra-fine grains of $100 \mathrm{~nm}$ in size covering approximately $60 \%$ of the volume and larger grains of about $2 \mu \mathrm{m}$ representing remaining $40 \%$ of the microstructure [5]. Retained R and B19' phases were observed locally in the large grains so it is not fully clear whether these results are representative and can serve as a solid basis for the microstructural scenario. In the present study, we tackle this issue by analysing the microstructure of homogeneous ultra-fine grained NiTi wires before and after functional fatigue. We systematically use selected area diffraction (SAD) technique in the transmission electron microscope (TEM) to sample a representative number of nanograins.

\section{Material and experimental procedures}

Commercial ultra-fine grained NiTi wires (nominal composition of 50.9 at $\% \mathrm{Ni}$ ) investigated in the present study were purchased from Memory Metalle, Weil am Rhein. Their processing route included hot extrusion at $650^{\circ} \mathrm{C}$ and subsequent cold work $45-55 \%$ during which the wire was drawn to a final diameter of $1.2 \mathrm{~mm}$. The processing was completed by a straight anneal treatment at $520^{\circ} \mathrm{C}$ for $60 \mathrm{~s}$ under load [6]. This procedure resulted in a homogeneous nano-grain microstructure throughout the wire cross-section with typical grain sizes ranging from 50 to $100 \mathrm{~nm}$. Mechanical testing was performed at $60^{\circ} \mathrm{C}$ using a dual-column Instron 5567 testing machine equipped with a temperature chamber. Wire specimens with a total length $50 \mathrm{~mm}$ were used $(30 \mathrm{~mm}$ gauge length and two times $10 \mathrm{~mm}$ for gripping). Differential scanning calorimetry (DSC) measurements were carried out in a TA Instruments $2920 \mathrm{CE}$ in the temperature range between -150 and $+150^{\circ} \mathrm{C}$ using cooling and heating rates of $5^{\circ} \mathrm{C} / \mathrm{min}$. Further details concerning the investigated alloy, mechanical testing and DSC measurements can be found elsewhere [3, 4].

TEM specimens were cut out of the as received and cycled wires and thinned down to $0.1-0.15 \mathrm{~mm}$ by grinding on emery papers (grit size down to 1000). Final double-jet polishing was performed at $19^{\circ} \mathrm{C}$ in a solution of $20 \mathrm{vol} \%$ nitric acid in methanol using Tenupol 5 from Struers. The microstructure of the wires was investigated at room temperature in three microscopes, Philips CM 12, Philips CM 20 and Tecnai $\mathrm{G}^{2}$ F20. Standard TEM techniques were employed including centred dark field (DF), SAD, convergent beam electron diffraction (CBED) and EDX analyses. Our SAD procedure aimed at statistically relevant data on the phase composition of the material before and after mechanical testing. Therefore, a selected area aperture of $6 \mu \mathrm{m}$ in diameter sampled from 3000 to 5000 nano-grains that resulted in ring-type diffraction patterns. The SAD images were processed by adaptive contrast control routines (ACC) with a setting that allowed a contrast enhancement for weak reflections [7]. The SAD images were further analysed using a program FIT 2D in order to assign $d_{h k l^{-}}$ spacings and corresponding $2 \Theta_{h k l}$ Bragg angles to individual $h k l$ reflections and to integrate diffracted intensities along the individual diffraction rings $[8,9]$. Special attention was paid to a calibration of the SAD patterns. The calibration takes the advantage of $\mathrm{B} 2$ austenite reflections that were observed in each SAD image. The TEM camera length $L_{S A D}$ of a particular SAD ring pattern is evaluated such that it minimizes the sum of squares

$$
S(L)=\sum_{h k l}\left(d_{h k l}-\frac{2 \cdot \lambda \cdot L}{D_{h k l}}\right)^{2}
$$

where $d_{h k l}$ are B2-plane spacings (calculated using a B2 lattice constant $a_{B 2}=0.3015 \mathrm{~nm}[10]$ ). $D_{h k l}$ are corresponding diameters of $\mathrm{B} 2$ diffraction rings measured in the SAD image and $\lambda$ is the electron wave length $\lambda(200 \mathrm{kV})=0.00251 \mathrm{~nm}$. The minimum condition for $S(L)$ yields

$$
L_{S A D}=\frac{1}{2 \cdot \lambda} \cdot \sum_{h k l}\left(\frac{d_{h k l}}{D_{h k l}}\right) / \sum_{h k l}\left(\frac{1}{D_{h k l}}\right)^{2}
$$

and we note that the sums in Eqs. 1 and 2 cover all the available B2 $h k l$ reflections observed in a particular SAD image (typically about 8 reflections). With the calibrated $L_{S A D}$, the non-B2 reflections presented in the pattern can be pinpointed with an accuracy better than $1 \%$ as far as the corresponding plane spacings and/or Bragg angles are concerned. 


\section{Results and discussion}

\subsection{Microstructure of as received wires}

A low magnification TEM micrograph in Fig. 2 documents characteristic features observed in the wire microstructure before functional fatigue experiments. Ultra-fine B2 austenite grains with typical sizes in the range of $50-100 \mathrm{~nm}$ represent more than $95 \%$ of the specimen volume. The remaining volume fraction is occupied by two types of secondary phases. Less than $1 \%$ of the volume represent elongated TiC precipitates (one particle together with its corresponding SAD inset is shown in Fig. 2) that are aligned with the extrusion and wire drawing direction. Drop-type pores are often located at the ends of the TiC precipitates. Moreover, these precipitates are frequently associated with long tail-like regions delimited by a faint bright field TEM contrast. The elongated tails extend up to $4 \mu \mathrm{m}$ from the central TiC particle and their volume fraction can be estimated as 4-5\%. SAD diffraction (see inset in Fig. 2) combined with chemical EDX analysis confirmed that the tails are composed of the $\mathrm{Ni}_{3} \mathrm{Ti}_{2}$ phase. Results of one representative EDX line scan across the $\mathrm{Ni}_{3} \mathrm{Ti}_{2}$ tail are summarized in Fig. 3. Next to the $\mathrm{Ni}_{3} \mathrm{Ti}_{2}$ phase no other Ni-rich precipitates, e.g. the $\mathrm{Ni}_{4} \mathrm{Ti}_{3}$ phase, were observed in the wire microstructure, neither in the as received nor in the cycled states. It has been shown by Hara and coworkers [11] that the $\mathrm{Ni}_{3} \mathrm{Ti}_{2}$ crystal lattice can be either orthorhombic (in the range of temperatures up to about $60^{\circ} \mathrm{C}$ ) or tetragonal (at temperatures above $60^{\circ} \mathrm{C}$ ). The transition between the two $\mathrm{Ni}_{3} \mathrm{Ti}_{2}$ polymorphs is in fact martensitic in nature [11]. However, this martensitic transformation does not seem to explain the observed asymmetric evolution of the hysteresis loops during functional fatigue of NiTi wires. One reason is that the drop of the plateau stresses demonstrated in Fig. 1 is characteristic for the broader temperature range $[3,4]$ and is not limited only to temperatures above $60^{\circ} \mathrm{C}$ where stress induced martensitic transformation of $\mathrm{Ni}_{3} \mathrm{Ti}_{2}$ precipitates can be expected. Moreover, precipitates that would transform to the orthorhombic "martensite" upon loading at temperatures above $60^{\circ} \mathrm{C}$ would retransform to their tetragonal "austenite" upon unloading and thus there is no potential to account for the type of "mechanical memory", which has been frequently reported in the literature, e.g. [2-4].

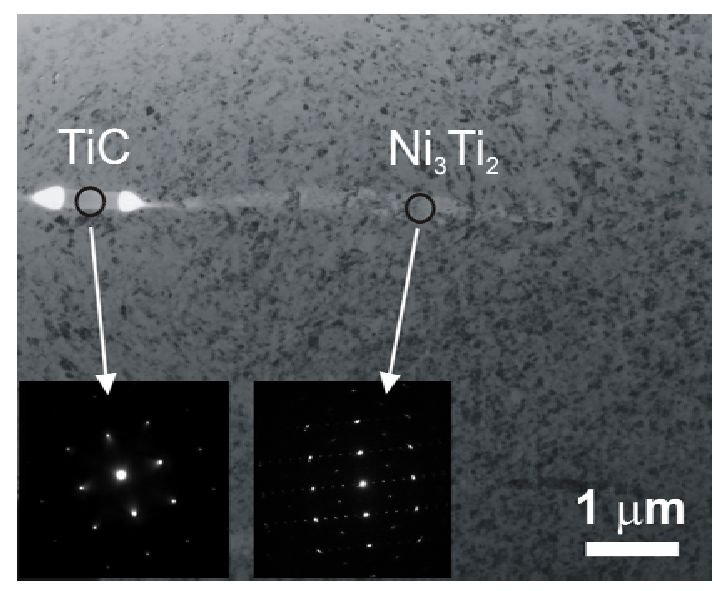

Fig. 2. $\mathrm{TiC}$ and $\mathrm{Ni}_{3} \mathrm{Ti}_{2}$ phases distributed in the ultra-fine grain B2 microstructure. Insets represent $<100>-\mathrm{TiC}$ and $<010>$ $\mathrm{Ni}_{3} \mathrm{Ti}_{2}$ zone SADs obtained from the indicated regions.
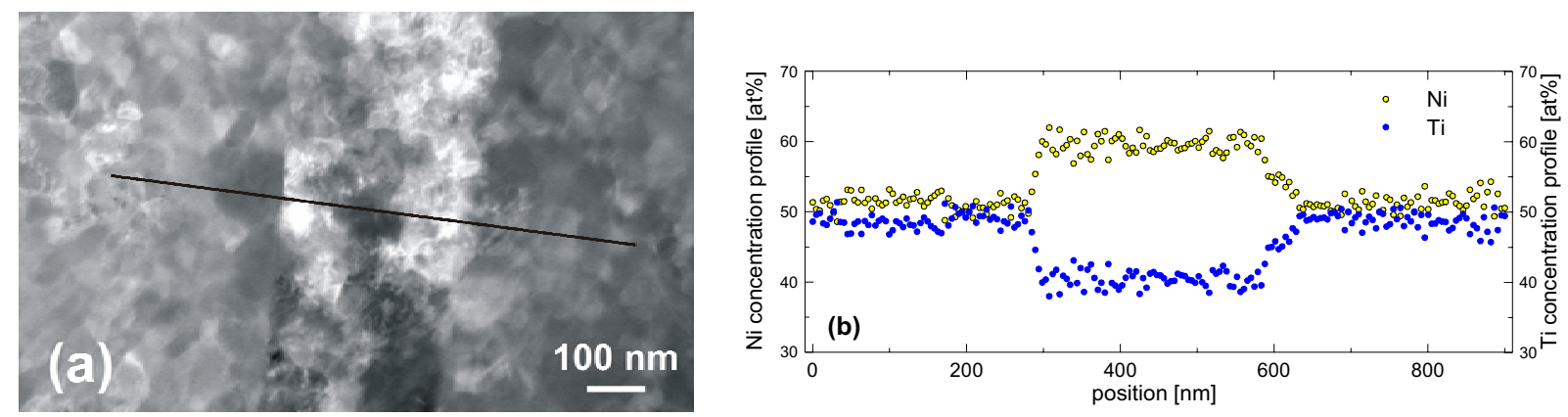

Fig. 3. EDX line scan across one $\mathrm{Ni}_{3} \mathrm{Ti}_{2}$ tail in the as received state of the NiTi superelastic wire. (a) HAADF image with the line indicating the position of the scan, (b) corresponding elemental line scan. 


\subsection{Misorientations of B2 grains}

Since the increase of dislocation density during functional fatigue is rather mild in nano-grained nicrostructures [5], there is an interest in the evolution of the character of grain boundaries caused by the loading and unloading cycles. Local internal stresses associated with the grain boundary structure may well contribute to the stabilization of R and B19' martensite nuclei and thus rationalize the observed transition of the mechanical properties. In Fig. 4 we present results of a preliminary analysis for the as received wire using a small probe CBED technique (Fig. 4a) and a DF method (Fig. 4b). The B2 cubic lattice orientation in four grains shown in Fig. 4a is represented by the cube projection method [12]. These orientations were evaluated using routines that are similar to EBSD procedures used in recent scanning electron microscopy (SEMs) [13]. Output data of the calculations in terms of the misorientation angles and corresponding rotation axes are summarized in Table 1. We note that the misorientation angles in Table 1 are presented after mapping to the fundamental domain of the Frank-Rodriguez space [14]. These data suggest that, except the boundary 1-2 between grains 1 and 2, the other three boundaries are of a high-angle character. It is also important to underline that, due to the ultra-fine grain size, statistically more relevant measurements based on a representative sample size of grains are still not possible even with advanced SEM EBSD facilities.

The dark field image in Fig. $4 \mathrm{~b}$ documents a microstructure in a TEM foil cut out of the wire after 80 loading-unloading cycles performed at $60^{\circ} \mathrm{C}$. The image was formed with a reflection encircled in the inset SAD pattern. This micrograph clearly shows that there are local volumes in the microstructure where orientations of small B2 crystallites are similar and, consequently, these crystallites are separated by only small angle dislocation boundaries that do not cause strong contrast changes. Some of those larger regions may correspond to former B2 grains of the microstructure before the final drawing and straight annealing operations. The preliminary results presented in Fig. 4 thus reveal that a broad variety of grain boundaries exists. Further work is needed to collect statistically more relevant data on the grain boundary character in the as received as well as in the cycled stated of the superelastic NiTi wires.
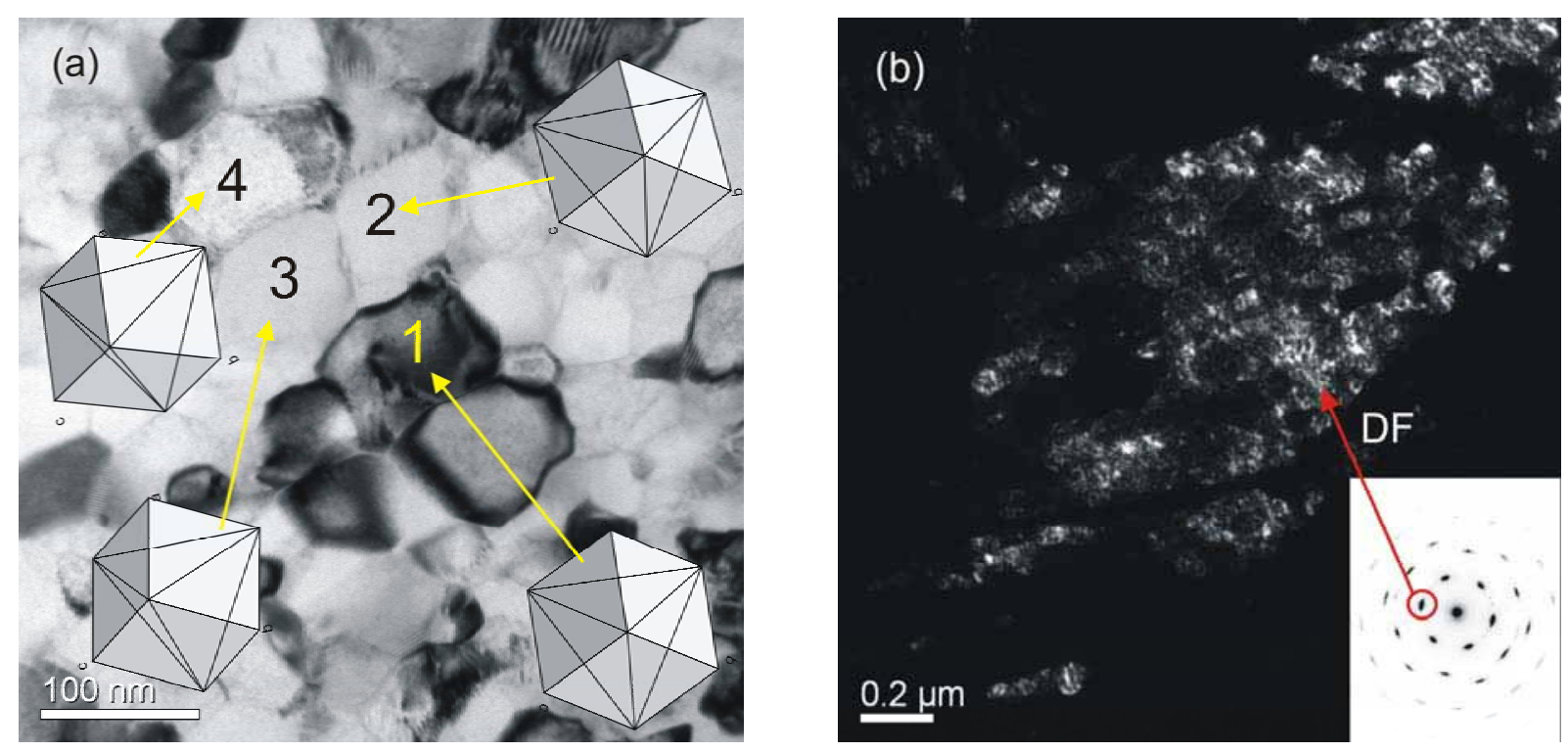

Fig. 4. Misorientations of B2 nano-grains characterized using small probe CBED and DF techniques for (a) as received wire and (b) wire after 80 superelastic cycles at $60^{\circ} \mathrm{C}$.

Table 1. Grain boundary characteristics, see Fig. 4a.

\begin{tabular}{|c|c|c|c|c|}
\hline Grain boundary & $1-2$ & $1-3$ & $2-3$ & $3-4$ \\
\hline Rotation axis & {$[-0.36,0.75,0.55]$} & {$[0.93,-0.23,-0.29]$} & {$[0.85,-0.35,-0.39]$} & {$[-0.02,-0.36,0.93]$} \\
\hline Rotation angle $\left[{ }^{\circ}\right]$ & 5 & 19 & 23 & 11 \\
\hline
\end{tabular}




\subsection{Selected area diffractions}

Here we present results of the systematic SAD study focused on the phase composition of as received wires before mechanical testing (Fig. 5a) and on the phase composition of a wire after 80 loading-unloading cycles performed at $60^{\circ} \mathrm{C}$ (Fig. 5b). As mentioned in the section 2, a relatively large selected area aperture has been used to sample a representative number of nano-grains. The individual diffraction experiments were performed in about seven different locations of three different TEM foils such that more than 20 SAD ring-type diffractions were obtained for each of the two material states. A factor that considerably complicated the evaluation was the presence of the $\mathrm{Ni}_{3} \mathrm{Ti}_{2}$ phase. Since many lattice plane spacings of the $\mathrm{Ni}_{3} \mathrm{Ti}_{2}$ phase are very close to those of either R or B19', the interference of these elongated precipitates masks expected weak reflections of retained martensitic phases even in carefully calibrated images. Therefore, approximately half of the SAD patterns that suffered from this drawback were eliminated and not considered for the evaluation. As an example, the SAD patterns in Fig. 5 fully represent the phase composition of the respective material states except the elongated $\mathrm{Ni}_{3} \mathrm{Ti}_{2}$ tails.

Only B2-phase reflections can be detected in the SAD pattern shown in Fig. 5a. This result is expected for the straight annealed Ni-rich NiTi wire that has not been subjected to mechanical cycling. There are indications of a texture in the SAD pattern of Fig. 5a, which was already reported earlier and described as a [111]-fibre texture for a similar type of wire [3]. On the other hand, the material state after the functional fatigue does exhibit some additional weak reflections situated round the main intensive $\{011\}_{\mathrm{B} 2}$ diffraction ring, see Fig. $5 \mathrm{~b}$. In order to display these weak diffraction rings more clearly and identify them unambiguously, slices of the SAD patterns near the $\{011\}_{\mathrm{B} 2}$ diffraction maxima were processed by the FIT 2D program. Results of these evaluations are shown in Fig. $6 \mathrm{a}$ and $6 \mathrm{~b}$ for the as received and cycled states, respectively. After a polar


Fig. 5. Representative SAD ring-patterns obtained for (a) as received wire and (b) wire subjected to 80 superelastic cycles at $60^{\circ} \mathrm{C}$. The indexes mark the $\mathrm{B} 2$ reflections.

transformation, the slices are unfolded and presented in Fig. 6 as line diffractions together with the intensity profiles resulting from the intensity integration along the former diffraction rings. A comparison of both intensity profiles clearly shows that, while a relatively smooth curve represents the intensity distribution for the as received material, shoulders marked 1,2 and 3 modify the $\{011\}_{\mathrm{B} 2}$ diffraction peak in the case of the cycled microstructure. The shoulders 1,2 and 3 correspond to the weak diffraction maxima and can be, with an accuracy of $1 \%$ or better, associated with the reflections $\{01 \mathrm{~T} 2\}_{R},\{0222\}_{R}$ and $\{12 \overline{3} 2\}_{R}$ of the R-phase.

The result shown in Fig. $6 \mathrm{~b}$ represents yet another evidence (see also [5]) that, in Ni-rich NiTi alloys subjected to the mechanical cycling, the R-phase is present in the microstructure within a temperature range where the R-phase would normally be not expected (Fig. 6a). This microstructural evidence is fully in line with results of DSC measurements that also confirm the stabilization of R-phase after superelastic cycling. Typical 

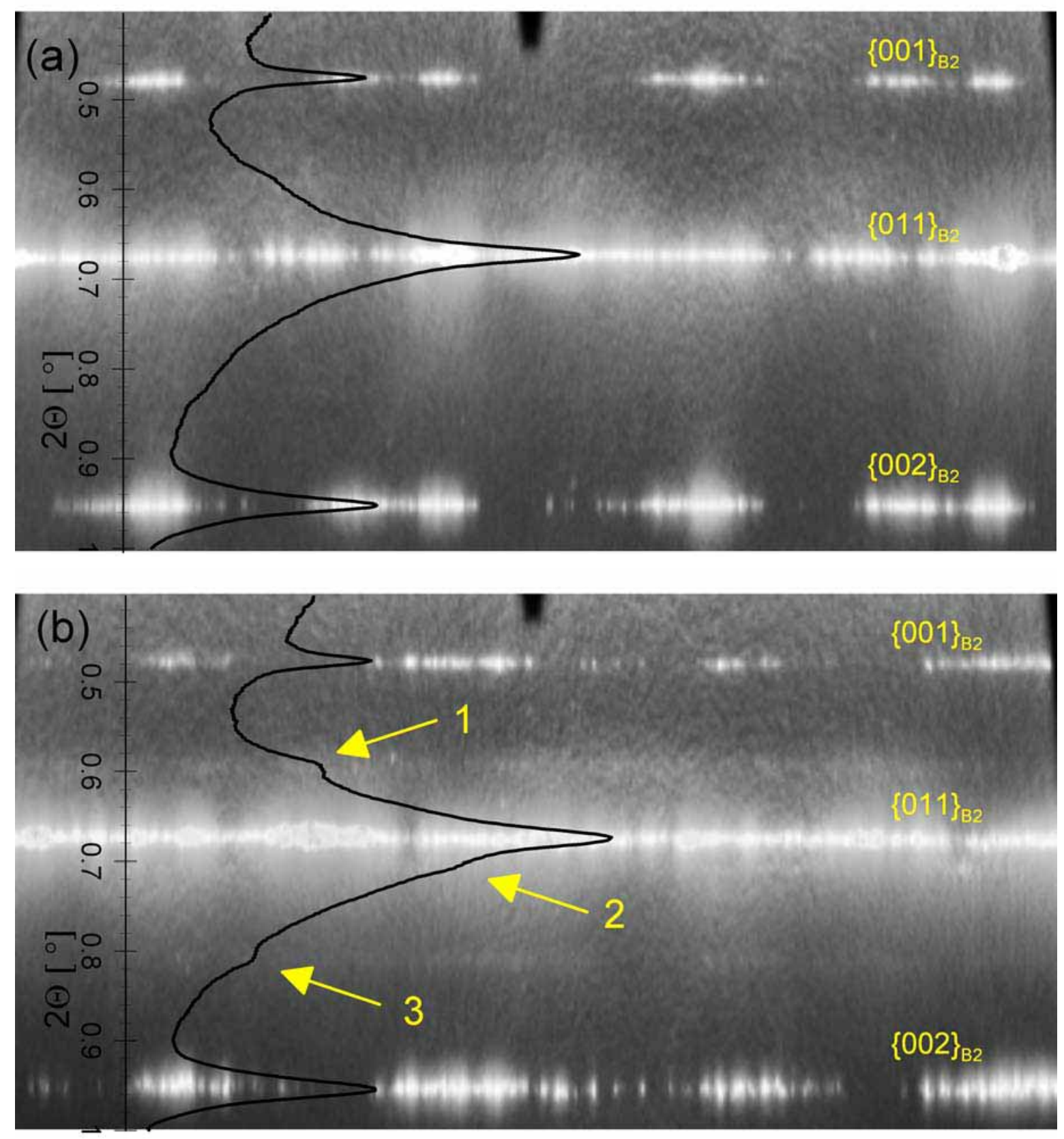

Fig. 6. Transformed and integrated SAD intensities of the Fig. 5 in the $2 \Theta-$-scale. (a) as received wire (see Fig. 5a) and (b) wire subjected to 80 superelastic cycles at $60^{\circ} \mathrm{C}$ (see Fig. 5b). B2 reflections are indexed. Shoulders marked 1, 2 and 3 in (b) indicate week R-phase reflections.

DSC charts obtained for the as received and cycled wires are shown in Fig. 7. It can be clearly seen that cycling increases three transition temperatures, namely $R_{s}, M_{s}$ and $A_{f}$, by more than $15^{\circ} \mathrm{C}$. It is interesting to note that the $\mathrm{A}_{\mathrm{s}}$ temperature is not affected by the cycling process and remains around $-40^{\circ} \mathrm{C}$ irrespective of the material state. As compared to the recent analysis of Ni-rich NiTi ribbons, where the R-phase stabilization was confirmed locally in larger grains [5], the microstructural data obtained in this study show that the stabilization effect is also important in the homogeneous ultra-fine grain microstructure of superelastic NiTi wires. In contrast to previous work and in spite of a relevant effort spent in the local analysis of individual nano-grains, it could not be clarified whether small nuclei of the B19' martensite also outlast the unloading part of the cycle and get stabilized in the unloading state of the wire.

The results of this and the earlier study [5] thus support the combined microstructural scenarios (see e.g. [3]) that account for the observed transition of hysteresis loops on the basis of retained martensitic phases stabilized by local internal stress fields of the B2 lattice defects like dislocations and/or grain boundaries. Results 


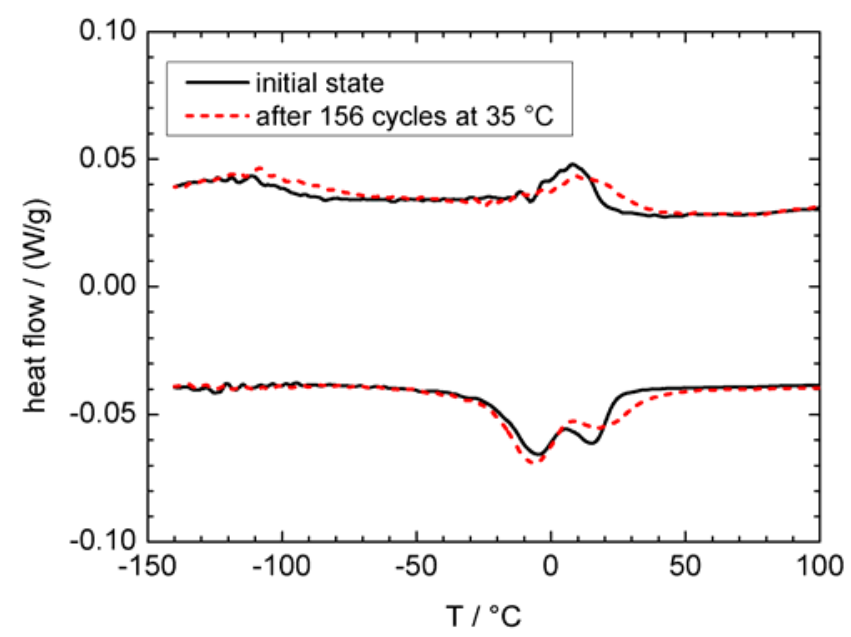

Fig. 7. Characteristic DSC charts of the as received wire (black curve) and the wire after 156 superelastic cycles at $35^{\circ} \mathrm{C}$ (red curve).

of recent molecular dynamics simulations point to the same mechanism and suggest that nuclei of martensitic phases with a typical size of $10 \mathrm{~nm}$ can be stabilized near the lattice defects with a relevant density [15, 16]. In accordance with an earlier suggestion [2], we expect that the B19'-phase nucleation controls the loading plateau stress. The gradual stabilization of $\mathrm{R}$ phase during cycling helps to reduce the B19' nucleation barrier (as compared to the direct B19' nucleation from B2 phase) and thus contributes to the gradual sinking of the loading plateau stress. An autocatalytic B19' nucleation in the ultra-fine grain microstructure [17] and its role in the evolution of the loading plateau stress still remains unclear and requires further investigation. On the other hand, since the direct transformation upon loading is never complete and some volume fractions of either B2 or R phase withstand the loading period, the re-transformation during unloading does not require the re-nucleation of the B2 austenite. Consequently, the unloading plateau stress is much less influenced by the microstructural evolution during cycling.

\section{Summary}

The microstructure of an ultra-fine grained superelastic NiTi (50.9 at \% Ni) wire has been investigated before and after mechanical cycling at $60^{\circ} \mathrm{C}$ using TEM techniques. The analysis confirmed that, after the straight annealing, the as received wires exhibit homogeneous distribution of fine B2 grains with typical sizes of 50-100 $\mathrm{nm}$ and different grain boundary characters. The alloy contains small volume fraction of TiC carbides and elongated $\mathrm{Ni}_{3} \mathrm{Ti}_{2}$ regions (volume fraction of 4-5\%) associated with TiC particles and aligned with the processing direction (extrusion and wire drawing). Our systematic selected area diffraction (SAD) study proved that the loading-unloading cycles promote the stabilization of $\mathrm{R}$ phase. It is suggested that the R-phase regions stabilized by internal stresses around dislocations and/or at grain boundaries facilitate the formation of B19' phase nuclei. The SAD results thus provide some support to the combined scenario that rationalizes the evolution of hysteresis loops during the NiTi wire cycling on the basis of B19' nucleation events that control the loading plateau stress.

\section{Acknowledgement}

The authors would like to acknowledge funding by Deutsche Forschungsgemeinschaft DFG through project A1 of SFB 459 (collaborative research centre on shape memory technology). A.D. received additional financial support from the Czech Science Foundation CSF under the contract no. 106/09/1913.

\section{References}

[1] S. Miazaki, in: Engineering Aspects of Shape Memory Alloys, edited by T.W. Duerig, K.N. Melton, D. Stöckel and C.M. Wayman (Butterworth-Heinemann, London, 1990), p. 394

[2] S. Eucken, T.W. Duerig, Acta Metall. 37, 2245 (1989)

[3] A. Yawny, M. Sade, G. Eggeler, Z. Metallkde. 96, 608 (2005) 
[4] J. Olbricht, A. Yawny, A.M. Condó, F.C. Lovey, G. Eggeler, Mat. Sci. Eng. A 481-482, 142 (2008)

[5] A.M. Condó, F.C. Lovey, J. Olbricht, Ch. Somsen, A. Yawny, Mat. Sci. Eng. A 481-482, 138 (2008)

[6] http://www.memory-metalle.de

[7] ACC - version 6.0, users manual (SOFO, Brno, 2008)

[8] A.P. Hammersley, ESRF Internal Report, ESRF98HA01T, FIT2D V9.129 reference manual V3.1 (ESRF, Grenoble, 1998)

[9] A.P. Hammersley, S.O. Svensson, M. Hanfland, A.N. Fitch, D.H. Häusermann, High Pressure Research 14, 235 (1996)

[10] Y. Kudoh, M. Tokonami, S. Miyazaki, K. Otsuka, Acta Metall. 33, 2049 (1985)

[11] T. Hara, T. Ohba, K. Otsuka, M. Nishida, Mat. Trans. JIM 38, 277 (1997)

[12] A. Dlouhý, G. Eggeler, Pract. Metall. 33, 1 (1996)

[13] A.J. Schwartz, M. Kumar, B.L. Adams, Electron Backscatter Diffraction in Materials Science (Kluwer Academic/Plenum Publishers, New York, 2000)

[14] F.C. Frank, Metall. Trans. A 19, 403 (1988)

[15] O. Kastner, G.J. Ackland, J. Mech. Phys. Sol. 57, 109 (2009)

[16] O. Kastner, private communication (Ruhr University, Bochum, 2009)

[17] T. Waitz, Acta Mater. 53, 2273 (2005) 